\title{
First Eyetracking Results of Dutch CoronaMelder Contact Tracing and Notification App
}

\author{
Jan-Willem van’t Klooster ${ }^{1(凶)}$, Peter Slijkhuis ${ }^{1}$, Joris van Gend ${ }^{1}$, Britt Bente ${ }^{2}$, \\ and Lisette van Gemert-Pijnen ${ }^{2}$ \\ 1 BMS LAB, Faculty of Behavioural, Management, and Social Sciences, University of Twente, \\ Enschede, The Netherlands \\ j.vantklooster@utwente.nl \\ 2 Department of Psychology, Health, and Technology, Faculty of Behavioural, Management, \\ and Social Sciences, University of Twente, Enschede, The Netherlands
}

Abstract. The coronavirus disease (COVID-19) has led to a global pandemic. Many countries are using contact tracing as one of the interventions to control virus transmission. In conventional contact tracing, index cases with positive test results are asked to provide contact information of close contacts who were at risk of acquiring infection from the index case, within a given time period before the positive test result. Mobile contact tracing apps can augment traditional contact tracing, as they exchange contact information wirelessly and semi-automatically, and allow for quicker warning of at-risk contacts.

However, little is known about user acceptance and perception of such contact tracing apps, or how people perceive the advice presented therein. In July 2020, the beta version of the official Dutch corona notification app was usability tested with participants from various age groups. This paper presents the results of the objective eye tracking measurements executed during these usability tests.

The results show that both health and process related information is easily overlooked in the app by participants. A closed-loop contact with the builders of the app allowed for a direct feedback channel and quick improvements during the development stage.
\end{abstract}

Keywords: Contact tracing app · COVID-19 · Eyetracking

\section{Introduction}

The coronavirus disease (COVID-19) has led to a global pandemic [1]. Many countries are using contact tracing as an intervention to control virus transmission. In conventional contact tracing, index cases with positive test results are asked to provide contact information of those close contacts, who were at risk of acquiring infection from the index case within a given time period before the positive test result. These contacts are then traced by health authorities and informed, warned, quarantined, and/or tested. Health authorities had to scale up their contact tracing staff extensively to execute this 
labor-intensive task. However, index cases may not remember all their close contacts, or do not know them (e.g. in public transport). Mobile contact tracing apps can augment traditional contact tracing, as they exchange contact information wirelessly and allow for quicker warning of contacts.

Yet, so far only little is known about user acceptance and perception of such contact tracing apps [2] and how people perceive advice presented by such apps [3], or whether they understand the exposure logging mechanism. After it was decided that the Dutch government will develop such a contact tracing app, it was therefore usability tested with participants from various age groups. The hypothesis is that such tests allows the app to be improved in an early stage and better fit these heterogenous groups, increasing in the end the acceptance by the public.

This paper specifically presents the results of the objective eye tracking measurements executed during these usability tests. Our research questions were: what can we learn from using eyetracking in usability testing an early covid notification app, and how can the results be used to improve the notification ecosystem. This paper aims to present the results from the eyetracking usability test, and demonstrate the value of such methods in this area as an addition to qualitative, opinion based usability methods.

The rest of this paper is structured as follows. Section 2 briefly introduces the Dutch corona notification app and the working of this app. Section 3 describes the evaluation method. Section 4 discusses the eye-tracking results and Sect. 5 presents conclusions.

\section{Corona Notification App}

The Dutch 'CoronaMelder' corona notification app is a smartphone application built by the Dutch Ministry of Health. It is using Google and Apple's exposure notification framework (GAEN) [3]. This is a decentralized framework that allows modern smartphones to anonymously collect and log encounters with other smartphones at close range, and warn their users in case identifiers of other phones were later associated with cases having a viral infection as confirmed by a health authority. When turned on, the notification app tracks back encounters for 14 days for other app users at close range for $15 \mathrm{~min}$. This is a good indication for exposure risk. In case the anonymous identifiers of positive cases available are matched in the local exposure log, the user gets an anonymous notification of risk of exposure, and advice on what to do: stay home, and get tested, if symptoms apply. If an app user is tested positive by the health authority themselves, they can notify other app users with the help of and confirmation by the local health authority.

Because of the Bluetooth low energy technology used for measuring distance between smartphones, sensitivity and specificity are around 70-75\%. It is nevertheless believed that contact tracing technology is beneficial in fighting the pandemic [2, $4,5]$ and their implementation is advised by multiple disease control expertise centra across the world [6]. Tracing apps, due to their nature, allow for much faster notification of possibly infected encounters as compared to traditional contact tracing methods, i.e., calling contacts by phone by the public health authorities. However, an internet connection and a relatively new smartphone is needed by the end users for tracing apps. 


\section{Methods}

The present study consisted of scenario-based usability tests of CoronaMelder with think aloud method, interview questions and a questionnaire. The usability tests took place between June 29 and July 3, 2020. A beta version of the app was tested using smartphones with iOS (version 0.1, build 172) and Android (version 0.3.1, build 107). The study was conducted in the DesignLab of the University of Twente. The BMS Lab protocol for corona-safe human-related research that was followed, was approved by the Executive Board of the University of Twente. The study was approved by the University Twente's Ethical Committee under nr. BCE200953.

\subsection{Participants}

Participants were recruited via convenience sampling by the authors, with a focus on elderly and younger people.

The usability tests were conducted individually. Six participants wore Tobii eye tracker glasses for gaze analysis [7]. Prior to the test, the nature and purpose of the study was explained and permission for participation was obtained through an informed consent form that was signed by the participants.

The tests started with general questions about the impact of the pandemic on participants' lives and about what they have already heard about COVID-19 apps. Thereafter, each participant conducted 4 scenarios on the app, which represent actual use of the app while they were asked to think aloud. These scenarios are as follows:

1) Introduction to the app: In this scenario, the app was shown in the app store and additional information about the app could be read.

2) Onboarding and activation of the app: In this scenario, operation of the app is explained through onboarding steps in the app itself. Participants learn about the content of the app and confirm the right permissions to use the app (allow the app to use Bluetooth and to send notifications).

3) Receive notification: In this scenario, the participants receive a notification from the app about their increased risk of infection, because they have been in in close contact to an individual who had tested positively for the coronavirus.

4) Sharing keys (telephone conversation with PHA): In this scenario, participants were asked to imagine that they have been tested positive on coronavirus recently. During the scenario they receive a phone call from the PHA, in which participants are asked about their symptoms and receive help with uploading the authorisation key for the contact tracing and to warn the smart phones with whom they were in contact with.

After finishing the scenarios, final interview questions were asked about participants' attitude towards the app and their willingness to use the app. 
Additionally, the UEQ-Dutch [8] was filled in. The interview and questionnaire aspects are outside the scope of this article. This paper focuses on the eye tracking study, as the other aspects will be reported in a separate article.

\subsection{Eyetracking}

Tobii AB Eye tracker Glasses 2 were used in this study. These glasses can provide objective data regarding gaze behavior in ambulant situations. Our goal in using them is to obtain insight into navigation behavior, search behavior, and the division of attention over text and visual aspects of the notification app [7].

The participants were measured during the execution of the abovementioned four scenarios. As such, the measurements record the first behavior of the participants that have no prior experience with the test object. The measurements were executed in 2 youngsters (secondary education), 2 youngsters (vocational education) and 2 adults.

\section{Eye Tracking Results and Interpretation}

Below results reveal both heatmaps and gazeplots. We present 2 different kinds of heatmaps. The first heatmap shows how long a person looks at a certain aspect. The second kind shows how often a test subject looks at a certain aspect.

The gazeplot shows the order in which the viewing behaviour occurs. In these plots, the size of the circle indicates the relative time duration of the viewing behaviour on that particular plave, and the numbers in the circles indicate the order of viewing. The color is indicative for the participant.

In the Fig. 1 gazeplots, the opening screen on the app is shown, which is visible after downloading and installing it. The opening screen of the app allows users to check that the GAEN mechanism is active ('de app is actief' translates to 'the app is active').

It allows users from top to bottom to select 4 menu item options to (i) get more information about the app; (ii) get information on what to do in case of an risk-of-exposure notification; (iii) get details on how to obtain a covid test; (iv) share an authorization key to notify other users in case the user had a positive lab-confirmed PCR test for covid-19.

The gazeplots show that participants pay significant attention to the image in the middle of the screen. The participants look at the image, and get back to that after scrolling and reading the menu items. Although the users have different behaviour, they look at both the image and the bold texts. 
A relatively large part of the viewing time is devoted to looking at the image, in which an animation of a person on a bicycle attracts attention. This happens at the cost of reading the menu item explanations an understanding the structure. The app screens in Fig. 2 show that although there is attention for the text of the menu items, attention also goes to the visual components of the people composition. Before the fold attention goes to the visual aspects; after scrolling to the clickable menu options.
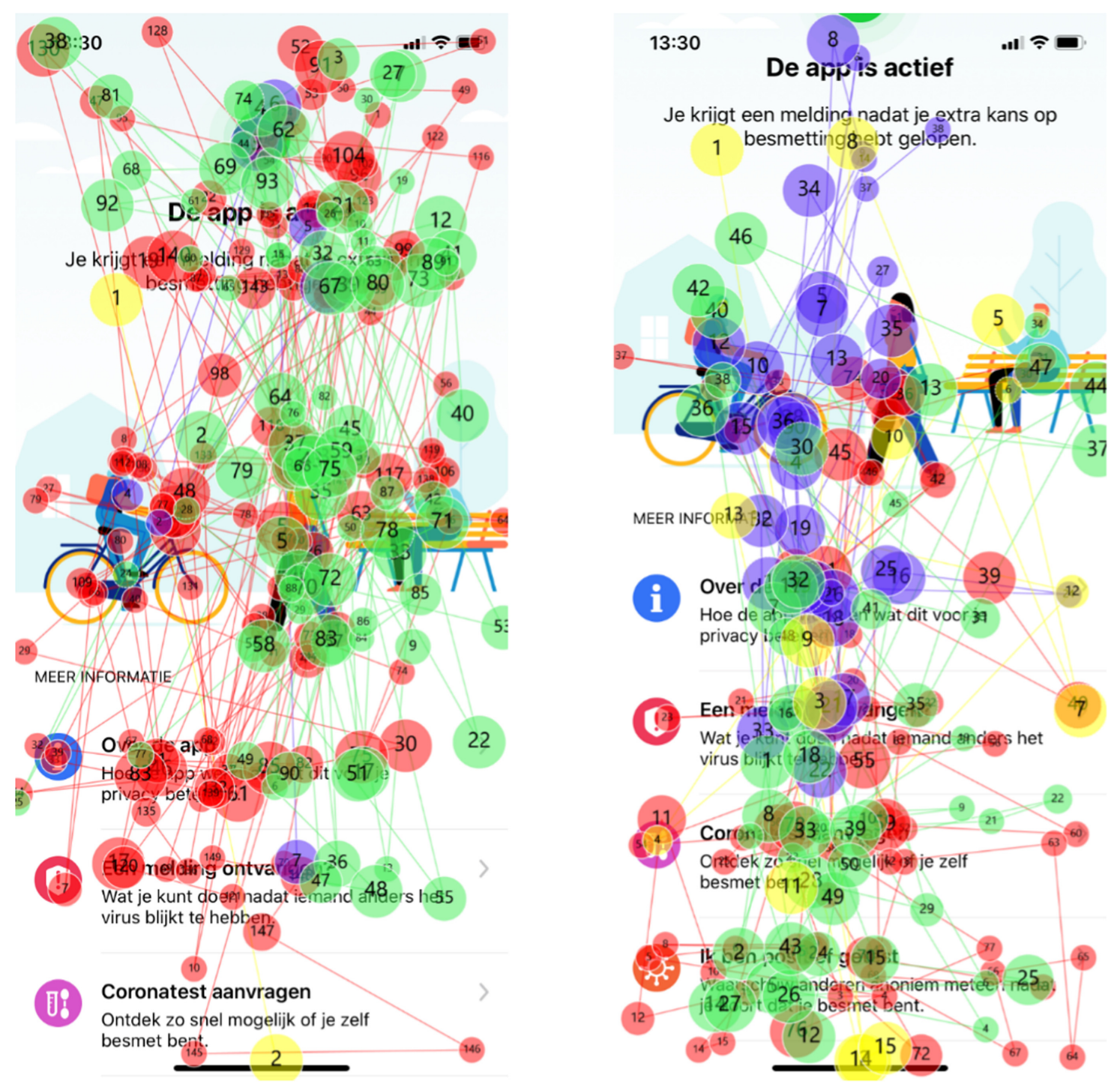

Fig. 1. Gazeplot (left) of the main menu before scrolling down $(n=4$ out of 6$)$. Gazeplot right) of the main menu ( $\mathrm{n}=4$ uit 6 ) after scrolling down (under the 'fold' in the screen). Each color represents one participant. Standard attention filter, cut-off $100 \%$ s. Tobii Pro Lab 1.142 (tobii, 2020). $\mathrm{n}=2$ out of six participants received an inactive screen, which is further discussed in [10]. (Color figure online)

Thus, the questions arises what the main goal of the introductory screen of the app is. If promoting understandability of the navigation structure is important, this is an argument for less visual animations and more salient texts. A pitfall of too much animation is visual saturation. 


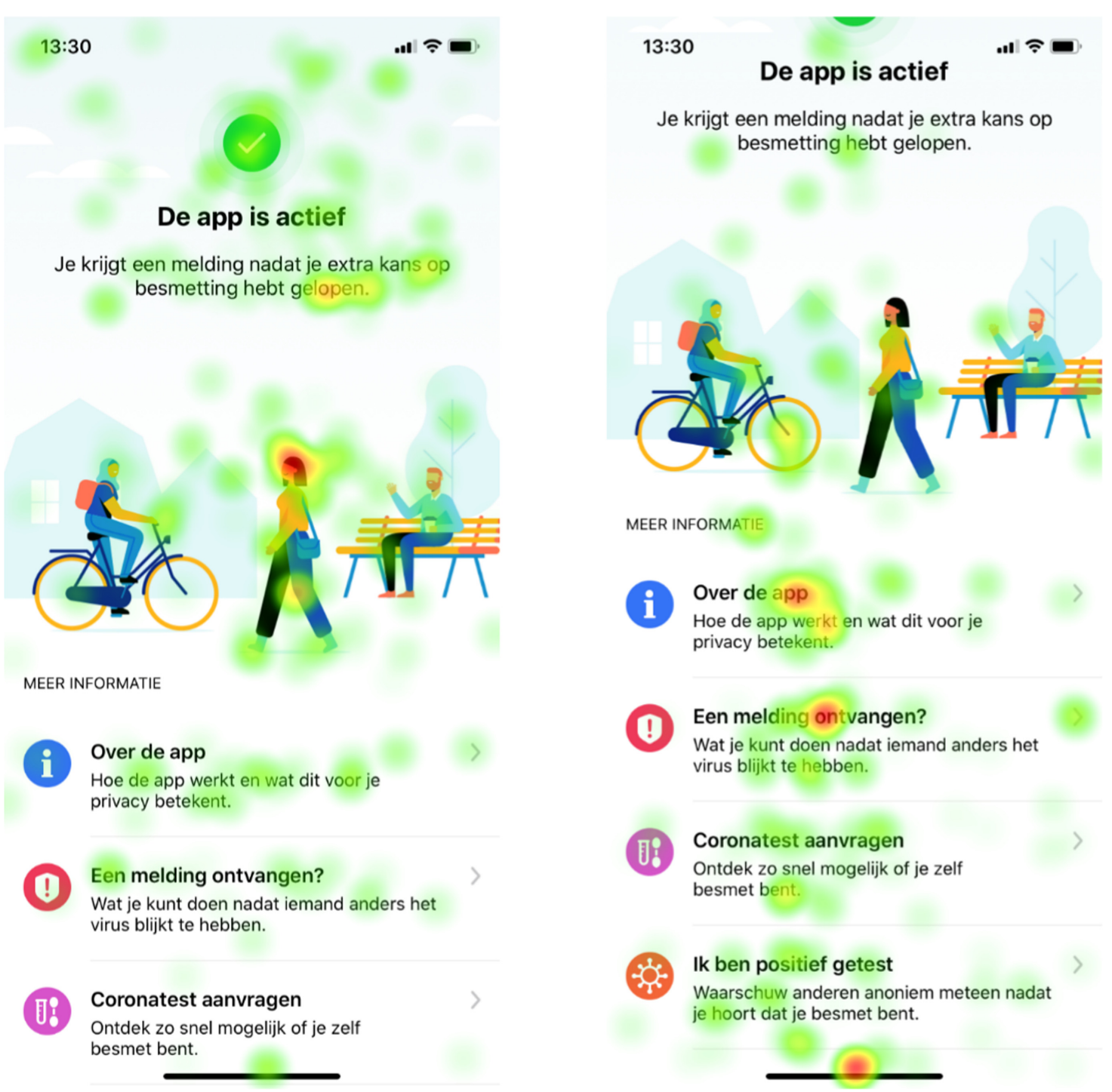

Fig. 2. Heatmap (left) of the main menu ( $n=4$ of 6 ) before scrolling down. Heatmap (right) of the main menu after scrolling down. More intense red means viewed longer, more green means observed shorter. (Color figure online)

If the app onboarding is not completed successfully, the app becomes inactive ('niet actief', shown in Fig. 3). This was caused by a software bug in the beta version, but can become a reality in the released app too. We clearly see participants are able to find solution to active it nevertheless, which is important. They look long and often at the 'instellingen aanzetten' (activate settings) button. They look more at the icons and search for solutions all over the screen, looking for visual cues. The prominent image at the top is not used here, so a reason could also be that participants are not yet visually saturated. 
When activating the app, a popup is used to enable exposure logging and notification. In Fig. 4, users are asked to accept turning on the GAEN framework to start logging encounters in the phone's logfile and to be able to receive exposure notifications. We observe that not all information is read, and that terminology ('COVID-19') receives more-than-average attention, both in time as well as in frequency. The title of this popup is barely read by these participants, yet terminology such as 'COVID-19' receives morethan-average attention. In addition, users also read texts that could have been blurred out because the popup is active.

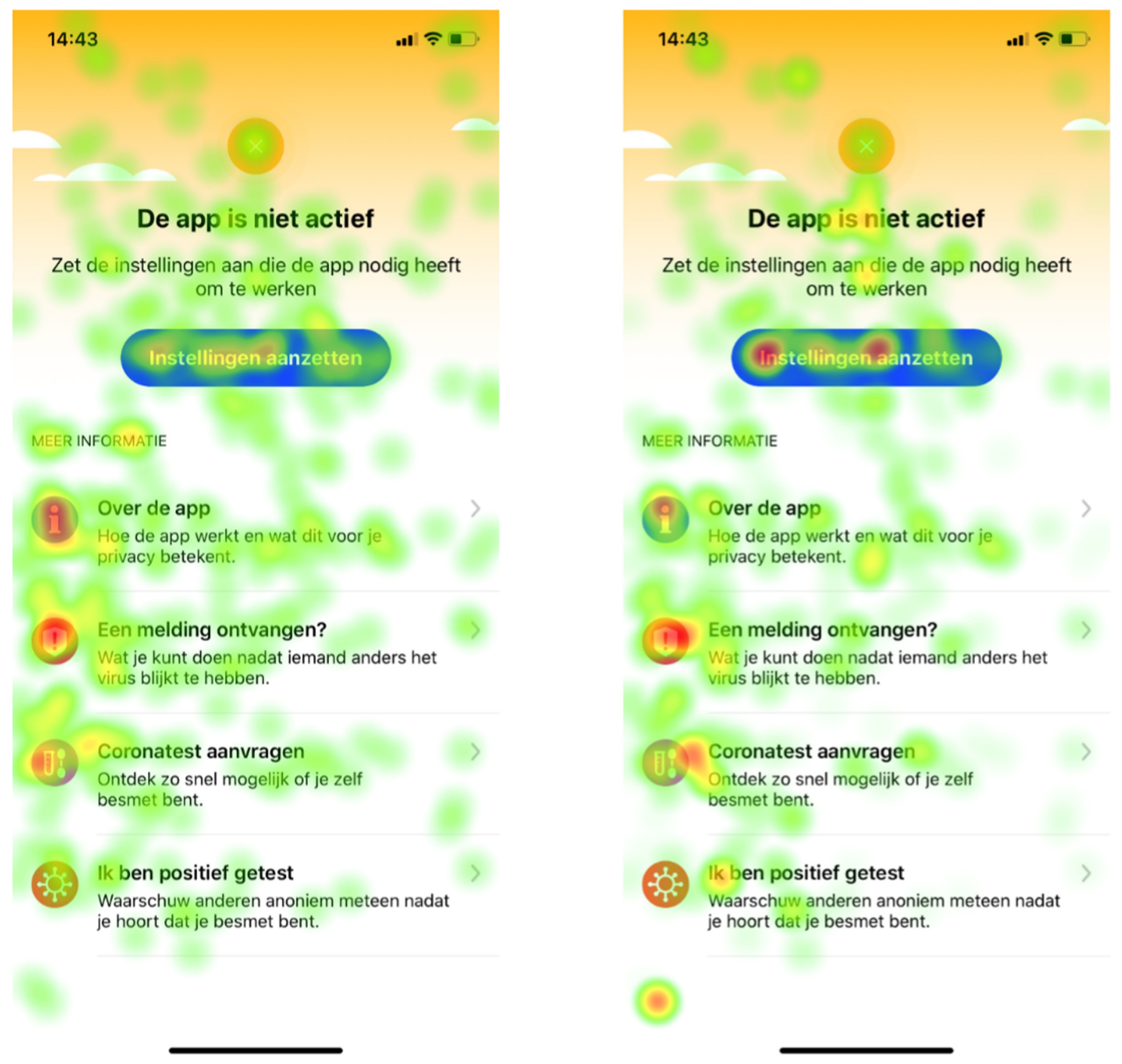

Fig. 3. Error in activating app, app becomes inactive and will not keep track of exposures ( $\mathrm{n}=$ 2 out of 6 , the 2 other test subjects). Left: Red means watched more frequently, green less often. Right: red means watched longer, green shorter time. (Color figure online)

It is not uncommon that users do not read all terms in a popup, neither so in healthrelated cases as compared to general use cases. The popups are often simply too long. In addition, prior screens already explain the to-be-expected behavior in this particular case. This is an intertwine between the app and the operating system. It is advised to 
show the most important information about rights in the first steps, then the pop-up notification text size can be reduced.
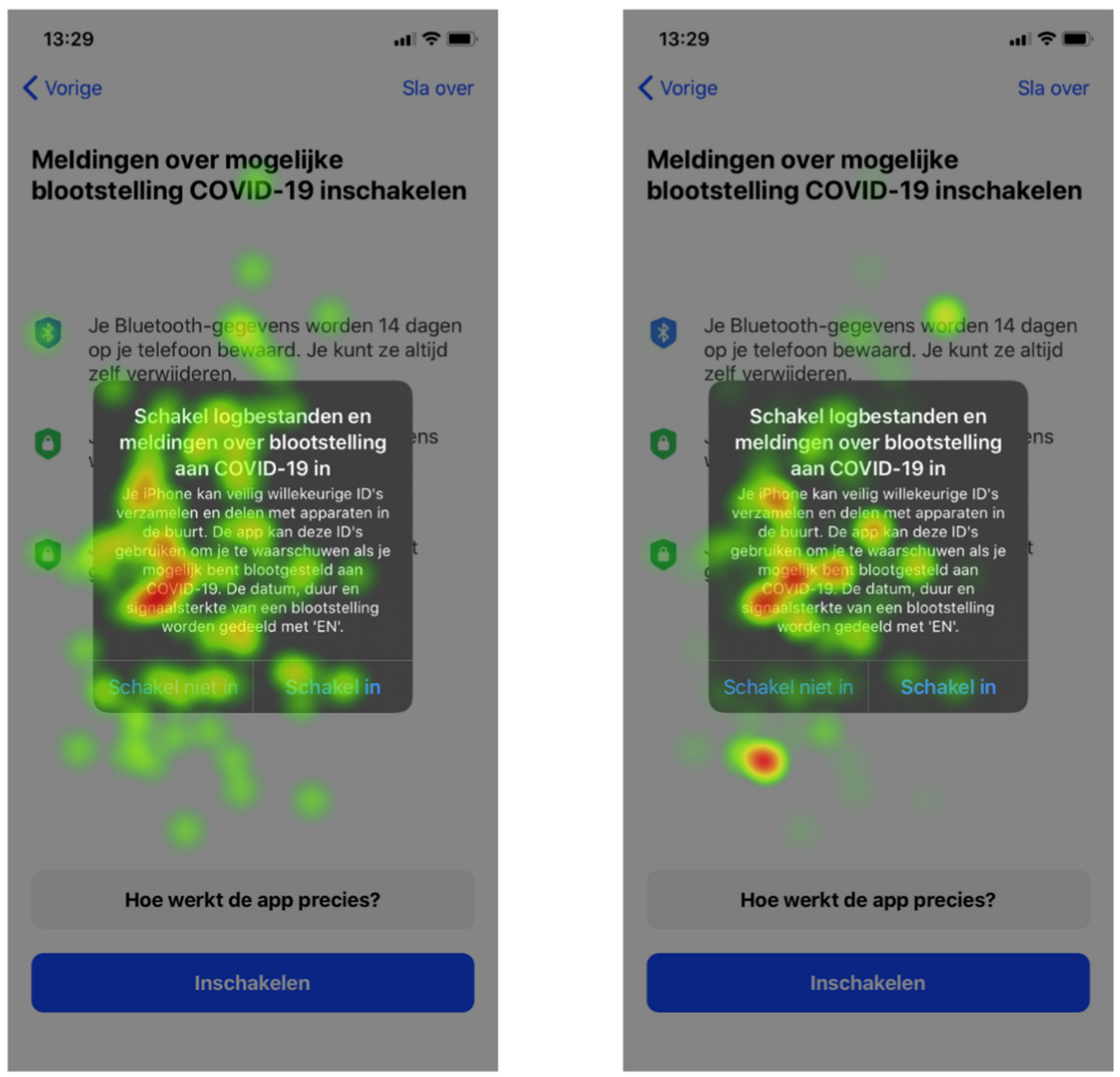

Fig. 4. Heatmap of the popup to enable GAEN Bluetooth framework $(n=3$ participants; others could not be adequately mapped) before main menu is accessible. Left: Left: Red means watched more frequently, green less often. Right: red means watched longer, green shorter time. (Color figure online)

\section{Conclusion}

This article presented the first eye tracking study of the Dutch corona notification app.

Eye tracking has shown to provide insights into the way covid-specific information is being perceived in notification apps. Both health related and process related information is easily overlooked by the participants. The attention to visual information is relatively large compared to attention to explanatory text in the virus-related informative screens. Theses screens are crucial in understanding the app correctly. 
Eyetracking also provides insight into the navigation and flow in using such an app, which in this case is especially relevant, given the covid- and notification mechanism related explanatory content. Moreover, eyetracking gives insight in the intensity and order of the users' perceptions. This showed that permission popup prompts in the app are important in validation steps in the usage process, yet were found to be improvable.

Improvement suggestions were shared with the builders of the app and the framework developers [9]. Importantly, a closed-loop contact with the builders allowed for a direct feedback channel and quick improvements during the app development.

A limitation of the study is that it had to be conducted under time pression because of the timeline for development set, and the need to come to countermeasures against the spread of the pandemic. This affected the number of participants, although for a usability study of a beta version testing with few users is known to already provide important input [10]. Nevertheless, it is important to keep evaluating and comparing contact tracing apps as they are implemented, as our recommendation for further research.

\section{References}

1. WHO COVID-19 (2020). https://www.who.int/emergencies/diseases/novel-coronavirus2019. Accessed 17 Feb 2020

2. Altmann, S., et al.: Acceptability of app-based contact tracing for COVID-19: cross-country survey study. JMIR Mhealth Uhealth 8(8), e19857 (2020)

3. Exposure Notifications Frequently Asked Questions (2020). https://covid19-static.cdn-apple. com/applications/covid19/current/static/contact-tracing/pdf/ExposureNotification-FAQv1.2. pdf

4. Gleeson, M., Neville, K., Pope, A.: The design and development of a digital contact tracing application to better facilitate the tracing of passengers in the event of a biological threat/ pandemic. J. Decis. Syst. (2020)

5. ECDC: E.C.f.D.P.a.C.: Mobile applications in support of contact tracing for COVID-19 - a guidance for EU/EEA member states (2020)

6. Jacob, S., Lawarée, J.: The adoption of contact tracing applications of COVID-19 by European governments. Policy Des. Pract. (2020)

7. Carter, B.T., Luke, S.G.: Best practices in eye tracking research. Int. J. Psychophysiol. 155, 49-62 (2020)

8. Hinderks, A., et al.: Developing a UX KPI based on the user experience questionnaire. Comput. Stand. Interfaces 2019(65), 38-44 (2019)

9. Rijksoverheid: Hoe is CoronaMelder getest? (2020). https://www.rijksoverheid.nl/onderw erpen/coronavirus-app/vraag-en-antwoord/hoe-wordt-de-corona-app-getest-voordat-dezebeschikbaar-wordt

10. Nielsen, J., Landauer, T.K.: A mathematical model of the finding of usability problems. In: Proceedings of ACM INTERCHI 1993 Conference, Amsterdam, The Netherlands, 24-29 April 1993, pp. 206-213. (1993) 